\title{
Design Representation in EDIF Version 300 and CFI Version 1.0
}

\author{
Hilary J. Kahn \\ Department of Computer Science \\ University of Manchester \\ Manchester M13 9PL, UK \\ e-mail: hkahn@cs.man.ac.uk
}

\begin{abstract}
The development of frameworks and integrated CAD solutions has, over the past ten years or so, led to the development of a number of standards which play a key role in the electronic product design and development cycle. Prominent among these are the Electronic Design Interchange Format (EDIF) and, more recently, the CAD Framework Initiative Design Representation (CFI DR). These two standards has been characterised by the use of a formal specification as an integral part of their development process. In both cases the published standard includes a specification in the form of an EXPRESS Information Model. CFI DR and EDIF also share another characteristic; they both cover the domain of electrical connectivity representation. In the case of CFI DR 1.0, connectivity is the main design aspect covered. EDIF Version 300 has a wider scope, including schematics as well as connectivity. In this paper, the information models of both CFI 1.0 and EDIF Version 300 are reviewed. The degree of overlap that exists between them is examined and indications given of a common or 'core' information model from which future versions of CFI and EDIF may be derived.
\end{abstract}

\section{Introduction}

It has been clear for a number of years that there is value, both from a theoretical standpoint and from a pragmatic point of view, in working towards the alignment of the underlying 'models' used by EDIF [1] and CFI DR [2]. Both standards relate to a fundamental part of the infrastructure of EDA systems. EDIF deals with the transfer of design information between EDA systems or from design system to manufacturing environment; CFI provides closely coupled communication between CAD tools and their support environments. Neither standard is targeted at end users, and both are independent of specific design flows or design stages.

Technical interchange of ideas has been fostered over the years by, for example, having a CFI representative on the EDIF Technical Committee during the development of EDIF Version 30 0 and by EDIF experts being involved in the review of the CFI DR information model. However, the climate has, until recently, not been right for the next step, the consideration of a formal technical alignment of EDIF and CFI DR. The catalyst for change, and hence for the study whose findings are reported here, was the decision by the EDA Companies consortium (EDAC) earlier in 1994 that they wished the various standards bodies and standards creators to make some positive move towards alignment. This strong push from the industry comes, of course, from the entirely pragmatic view point that it is not cost effective for the major EDA vendors to be involved in the development of a number of, possibly competing, standards. The 
cost is high in terms of money and manpower to maintain competence in different standards, and in terms of development and support effort to build compliant software.

As a result of the EDAC initiative, a series of technical meetings took place between Laurence Brevard of CFI and Hilary Kahn representing EDIF. The initial goal was to investigate the degree of overlap between EDIF Version 300 and CFI DR 1.0 and to advise the EDA companies whether or not a core or common model between the two standards was feasible. In the event, the result was not only the identification of areas of overlap but also an assessment of some of the ways in which maximal mappings between EDIF and CFI could be achieved and a proposal for the constituents of a core model. The inputs into this assessment process were the two information models and a technical report [4] which presents in great detail a review of the minimal mappings that are supported by the two basic models.

\section{The EDIF and CFI Models}

The information models of EDIF Version 300 and CFI DR both use EXPRESS Version N14 [3]. The use of the same modelling language in many ways eases the task of comparing the models. However, each model is written in a specific style which may, in part at least, be justified by the subtle differences in the EDA system domains to which they apply i.e. Programming Interface (PI) vs. file-based design transfer

\subsection{CFI DR 1.0}

The CFI 1.0 standard, published in 1992, includes an information model of design representation as part of the formal documentation. The model is small, consisting of seven schemas with a total of just over 20 entities (domain objects). The schema structure reflects the objects in the domain rather than broader aspects of the design domain, Hence, the schemas relate to: library, cell, net, port, instance and portInstance, as well as a unifying 'basic object model' schema. The goal of the model is the formalised specification of the domain covered by CFI DR; this is used as the basis for the PI function specifications which are the real 'product' in the CFI DR standard. CFI supports the representation of non-parameterised design.

The CFI DR model has a consistent style in terms of the representation of basic characteristics. For example, the names of entities have been chosen to fit in with the intended PI notation. So, in an EXPRESS class structure (supertype-subtype), the name of the supertype typically is the most commonly used term; the subtypes are then appended with additional characterising names. An example is illustrated in the model fragment in Figure 1.

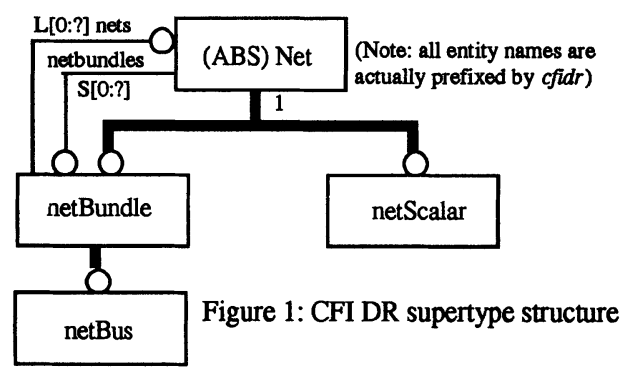

In the PI based on this model, access and manipulation functions exist for operating on the net object itself (e.g. cfidrNetDetachPort) and on its different subtypes (e.g. cfidrNetBundleInsertNet). 


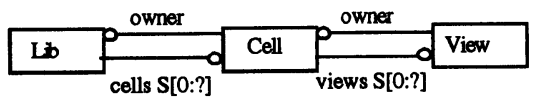

Figure 2: CFI owner attribute

Another style used in the CFI DR model is the explicit identification of 'owner' relationships. Although EXPRESS provides mechanisms which establish existence dependency (i.e. the INVERSE attribute), in the CFI DR model this is not used. Instead an explicit owner is defined as illustrated in Figure 2.

This mechanism is justified by the importance of the role played by the owner relationship in a PI. In CFI DR, there are a number of functions which explicitly manipulate the owner attribute (e.g. cfidrCellGetOwner, cfidrPortSetOwnerView) and the create functions (e.g. cfidrPortBundleCreatePortBundle) all have the owner attribute as a parameter. It should be noted that, although it provides the basis for the PI in terms of objects and relationships which must be supported, the CFI information model does not dictate the syntactic form of the CFI DR PI. That is determined by considerations of issues such as run-time efficiency and ease of use.

\section{$2.2 \quad$ EDIF Version 300}

The EDIF information model is much larger than that of CFI, mainly because it covers a wider area of design representation, including schematic diagrams and design configuration, as well as connectivity. In addition, as EDIF deals in static and in parameterised design representation, it is defined at two separate levels. EDIF Level 0 handles all the requirements for the transfer of non-parameterised designs and libraries; EDIF Level 1 adds to this the capability to represent frames and parameterised designs. Hence, the formal standard includes two separate information models; one for Level 0 , the other for Level 1. For the purposes of this discussion, only the Level 0 information model will be considered.

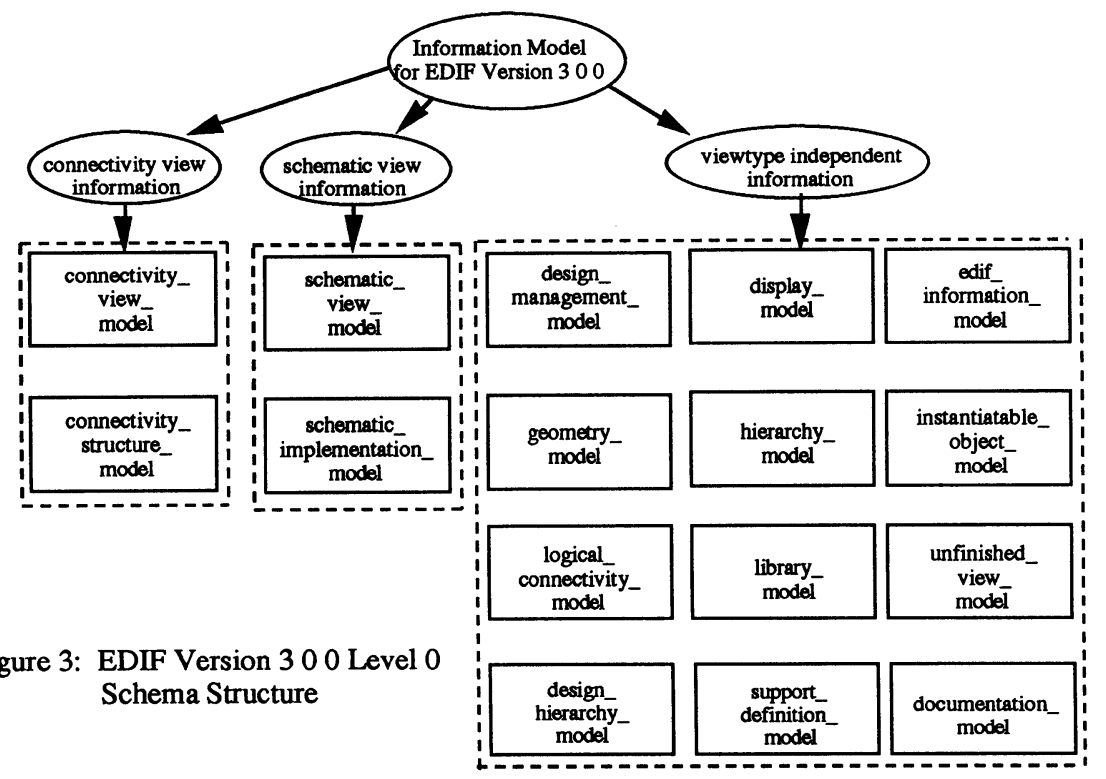


The Level 0 information model consists of 16 schemas divided into separate sub-domains. Some of the schemas are specific to the type of EDIF view being described; the majority of the schemas, however, cover information which is either common to more than one type of view e.g. the hierarchy_model schema, the logical_connectivity_model schema, or represents fundamental concepts e.g. the geometry_model schema.

Within the EDIF information model care has been taken to use consistent object naming conventions; these are typically directly reflected in the corresponding EDIF keywords in the syntax. However, just as the CFI information model does not dictate the form of the CFI DR PI calls, so the EDIF information model does not determine syntactic issues such as the underlying use of 'define before use' in the EDIF format or the Lisp-like lexical structure.

However, some fundamental relationships specified in the model are found reflected in the EDIF syntax. These include the concepts of 'containment' and 'uniqueness' in a given context. In EDIF, the relationship that establishes the existence dependency of an object on another object, is not established using the EXPRESS INVERSE construct; instead, the function 'belongs_to_one' is used. Here it is illustrated used in the context of a cell belonging to one library. Note also that the cells in a library are defined as a set.

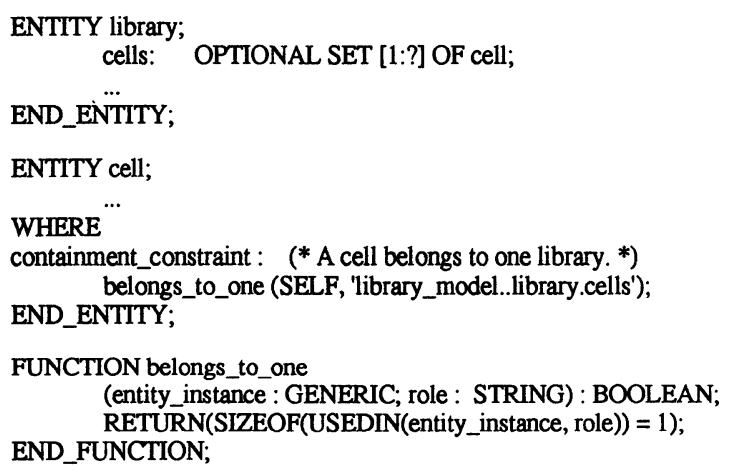

\section{Design Hierarchy}

Within EDA systems design information is typically held in some form of hierarchy. Where information is held in libraries, the natural form of the hierarchy is a folded definition hierarchy. In other words, each object (typically a 'cell') in the library is the definition of some design object. It is a two-level hierarchy description in which the object is defined strictly in terms of a network of instances of simpler objects. The hierarchy is always two level, regardless of whether the instanced objects are themselves hierarchical. See Figure 4.

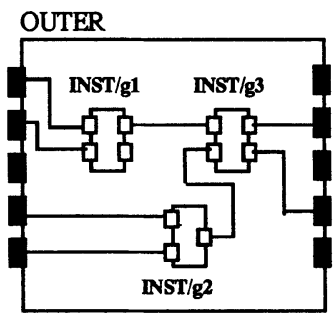

Figure 4. Folded Definition Hierarchy 
In both CFI DR and EDIF, this folded definition hierarchy is used for library object (e.g. cell) definitions. In EDIF, objects may be given properties and attributes when they are defined; those attributes and properties may be inherited when the objects are themselves used through the instantiation mechanism. In addition, EDIF provides for the overriding of the inherited attributes and properties at the point of instantiation. The inheritance and overriding of properties and attributes is not supported in CFI DR.

A complete design described in an EDA system may additionally be represented (possibly temporarily during processing) as an unfolded or expanded occurrence hierarchy. Where an unfolded occurrence hierarchy is not supported, a flat representation may be used instead. These forms of design occurrence representation are shown in Figure 5. They are not directly supported in CFI. However, in EDIF, there is a mechanism for configuring an unfolded design hierarchy and for annotating property and attribute values to the occurrences.

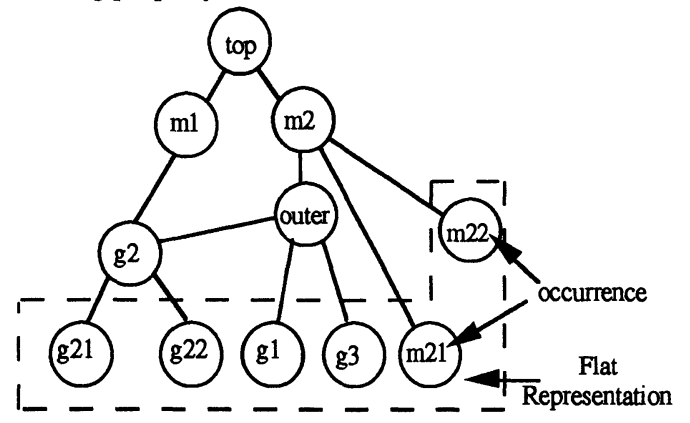

Figure 5: Unfolded Occurrence Hierarchy and Flat Representation

\section{The Goal of a Common Model}

Unless active steps are taken to avoid divergence, the development process for both EDIF and CFI will evolve as shown in Figure 6 (a). Each standard will resolve issues strictly within its own purview without regard to future coherence.

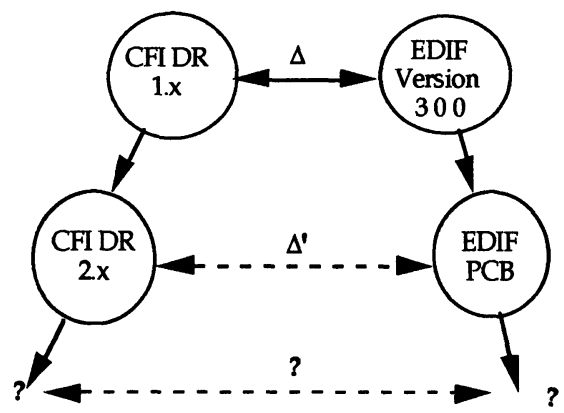

(a)

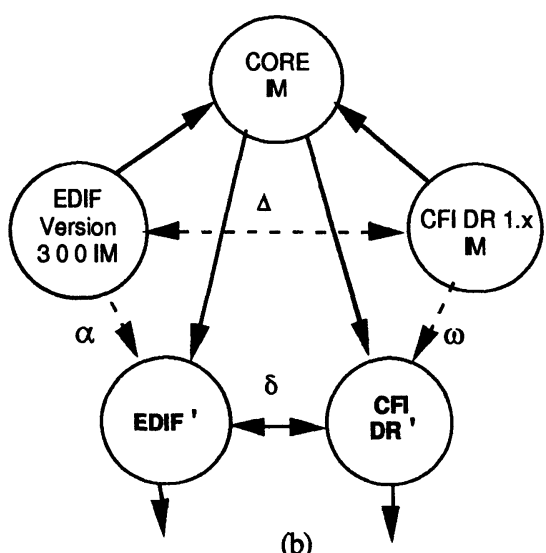

(b)

Figure 6: (a) The Current Situation (b) The goal of the EDIF-CFI alignment activity 
What is being sought by the EDA industry is a single underlying model from which the standards that they need to support have been derived. This is shown in Figure 6 (b), where the core information model is obtained by a reverse engineering process from the present EDIF and CFI models and then itself used to create future EDIF and CFI versions. The primary aim is to reduce the divergence from $\Delta$ to $\delta$. The intent is that $\delta$ should be minimised - and that, at the same time, $\alpha$ and $\omega$ should be kept as small as possible. This process encourages the future standards developments to take place in step with each other.

\section{Domains of Overlap}

The scope of EDIF Version 300 is connectivity, schematics and design configuration, together with the support of libraries and definition hierarchy; that of CFI DR is connectivity, libraries and definition hierarchy. There is clearly therefore a mismatch between the current coverage of the two standards. Should CFI DR find it appropriate to move towards coverage of those domains outside its present scope, it would seem natural that EDIF Version 300 would be a major resource from the information model point of view. In the area where overlap exists, that of connectivity and hierarchy, a deeper analysis is required. The following sections review some of the relevant aspects.

\subsection{Hierarchy}

In EDIF, the hierarchy is defined in terms of library $\rightarrow$ cell $\rightarrow$ cluster $\rightarrow$ view; in CFI DR only library $\rightarrow$ cell $\rightarrow$ view is used (as in EDIF Version 200 ). Furthermore, in EDIF, the object that is instantiated is the cluster whereas in CFI DR, it is the view. The issues therefore become: why did EDIF introduce the concept of cluster and is cluster a relevant concept in a core model?

The cluster introduced into EDIF allows all the cell representations (views, symbols) that share a common interface to be grouped. During any instantiation process a 'contract' is established between the instantiating environment and the instantiated object. In design terms, what is required is that the instantiating environment can relate to the interface (i.e. the ports) of the instantiated object. The local representation used to show this instantiation relationship in an EDA system may be the drawing of a symbol on the instantiating schematic page or a reference to the object from a language context. However, the true representation that will be used when the design hierarchy is traversed to produce the occurrence tree for a target process (such as simulation or the manufacture of the design) is selected by the requirements of that target process. This is supported in EDIF by instantiating a cluster and then allowing the design configuration process to select a suitable view from among those belonging to the given cluster. No equivalent mechanism exists in CFI DR although CFI does currently offer a view select mechanism which allows the describer of the selected view to be changed - but without any constraints on the suitability of the new view or its interface.

The characteristics of a cluster as described above are relevant beyond the domain of EDIF. It is therefore appropriate for the cluster to become a core model concept. Note that a benefit of cluster as a grouping mechanism for cell representations that have a common interface is that it is a step towards simplifying mappings between ports of different views.

\subsection{Connectivity: Logical and Structural}

In EDIF, a distinction is made between logical connectivity and structural connectivity. Logical connectivity captures abstract view-wide connectivity in terms of single-bit objects (which are called signals) linking single-bit ports. Any type of view which supports connectivity data includes logical connectivity. The logical connectivity is the basis for the 
structural connectivity which 'implements' it. Structural connectivity is expressed in terms of nets and busses, which may themselves be structured into sub-nets and sub-busses. Structuring into sub-nets and sub-busses allows implementation attributes, such as timing data and routing criticality, to be associated with parts of connections and point-to-point links. CFI DR currently supports the concepts of logical connectivity, but not of structuring.

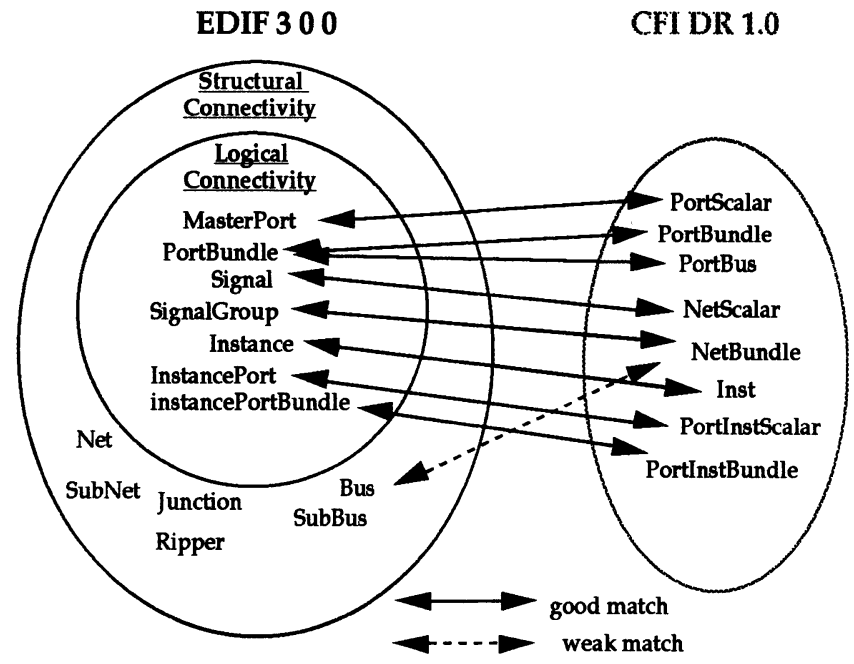

Figure 7: EDIF and CFI Connectivity Overlap

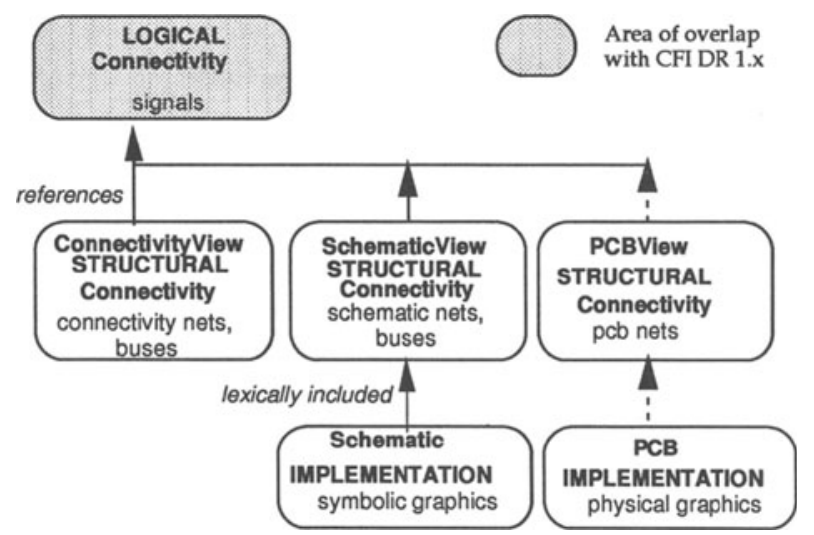

Figure 8: Relationship between Logical and Structural Connectivity in EDIF

In EDIF and CFI DR the fundamental connectivity supported in both models in strictly singlebit. In EDIF signals join ports; in CFI DR netScalars join portScalars. Both standards superimpose on this fundamental connectivity model facilities for representing wide connectivity. In EDIF, busses implement groups of signals (signalGroups) and are associated with 'wide' ports (portBundles); in CFI DR, netBundles group netScalars and are associated with portBundles. In neither case is connectivity actually established by the bus/bundle mechanism. In EDIF the existence of the wide connectivity objects is required to match the 
models commonly used in EDA systems and to support, for example, the drawing of bussedobjects on a schematic; in CFI DR, the wide objects provide a commonly used short-hand mechanism for manipulating grouped objects.

Figure 8 shows the general architecture used in EDIF to relate logical and structural connectivity. This approach supports extensibility of the domains covered, as is illustrated by current developments which are enhancing EDIF to include a PCB/MCM capability. These concepts are relevant to an EDIF-CFI core model, not only because they are already fundamental to EDIF, but because CFI is moving into new domains, including the PCB area and the incorporation of timing data.

\subsection{Naming Philosophy}

The study of the EDIF and CFI DR information models and reference manuals highlighted some interesting points about how names associated with objects are used in each standard. In order to understand what is required, it is necessary to recognise the purposes to which these object names may be put. Objects can be named in order to make them identifiable in a given context (i.e. they have a unique name) and object names are used as 'pointers' to allow objects to be referenced. In EDA systems, objects are also given names which the user selects - these user-defined strings allow the designer to annotate a design in a manner meaningful to him; in this context, name aliasing is also commonly found. Finally, objects may be given default names by the EDA system itself- a function that is needed where the user elects not to provide a name.

In EDIF Version 300 , a move was made away from the identifier and name overloading of EDIF Version 200 . As a result, the EDIF identifier is used exclusively for making an object unique in a given context and for referencing within the EDIF file. Support for user-defined names and for names supplied automatically by the EDA system is provided by a new nameInformation construct. In CFI, cfidrNamedObjectName establishes object uniqueness but object referencing is done using the cfidrObjectId (OID) which is the reference handle unique to a given CFI session. As with EDIF Version 200 , CFI provides no mechanism for capturing the user- or system-supplied names for an object although in some cases (see 5.4.1), overloading of the cfidrNamedObjectName mechanism is used to achieve a similar effect.

For a core model there is no need to define a single implementation mechanism for specifying object uniqueness in a context/session; CFI will continue to use the OID and EDIF will use the EDIF identifier. However, a mechanism for capturing the 'real' object name is required; a solution based on the EDIF nameInformation is suggested.

\subsection{Grouping Philosophy}

One of the few areas where EDIF and CFI DR deal with a single issue in different ways is that of grouping objects. A number of different types of object may be grouped: ports, signals, nets, instances. The reasons for forming these groupings are not always consistent. The grouping of ports and signals/nets are considered here.

\subsubsection{Grouping Ports.}

In EDIF, portBundles are formed by grouping existing ports and portBundles. PortBundles are defined in the cluster interface. A port/portBundle may be included in more than one portBundle but a port or portBundle can appear at most once in a given portBundle. 
LocalPortGroups in EDIF are handled in a similar manner to portBundles. In CFI DR, cfidrPortBundles provide a defining context for the cfidrPorts and cfidrPortBundles they contain. Hence a cfidrPort/PortBundle can be in at most one cfidrPortBundle.

Despite the apparent difference in the portBundle in each standard, the fundamental fact remains that in both standards the real connectivity objects are the single-bit ports and their single-bit connections, as noted above. It is therefore necessary to examine what each standard gains from the mechanism adopted. The EDIF model directly reflects the fact that the ports are the atomic objects and portBundles are convenient groupings. In CFI, the fact that a portBundle defines the ports it contains supports a specific requirement for the interface operation of 'connect by name'. As the cfidrPortBundle provides a name scope for the objects that it contains, a shorthand operation that causes all objects with the same 'name' to be connected is possible. Clearly, this relies on the overloading of cfidrNamedObjectName noted above.

It is therefore proposed that a compromise model be adopted as the core representation. The use of the EDIF-like mechanism for grouping existing ports into portBundles will be maintained although a constraint to ensure that a port is included in at most one portBundle may be agreed. In addition, rather than rely on the overloading of cfidrNamedObjectName to associate a name with the composite object members, a new attribute designed specifically to support member naming is likely. Provisionally, this is referred to as the localNameTag and its effect will be to give an object grouped by a portBundle a 'name' unique to its use in that context.

\subsubsection{Grouping Signals and cfidrNets.}

Here the difference in the grouping mechanisms used in EDIF and CFI is minimal. In both cases, the grouping construct (signalGroup or cfidrNetBundle) groups existing single bit or grouped objects. In both cases, too, a signal/cfidrNet may appear in more than one group and more than once in a given group.

The mechanism suggested for the core model is similar to (but less constrained than) that proposed for ports above. In other words. the grouping entity will group existing objects; in addition, objects inside a group may optionally have an associated localNameTag attribute to support membership naming.

\subsubsection{CFI Bus Objects}

In CFI DR there are objects called cfidrNetBus and cfidrPortBus which are formally subtypes of the cfidrNetBundle and cfidrPortBundle respectively. These objects have a relatively weak definition in CFI DR. Their main distinguishing feature is that they can be treated as indexed objects i.e. their members may be addressed by index. EDIF has no equivalent subtype for signalGroup or portBundle, but it does capture knowledge of the bus-indexing mechanism used in an EDA system through the nameInformation construct. On the assumption that the core model will adopt the EDIF-like nameInformation mechanism as suggested above, it is likely that a constrained form of the mechanism already in place in nameInformation will be used in the core model to provide CFI with the equivalent of the cfidrNetBus and cfidrPortBus

\section{Summary}

This paper has addressed some of the issues that arose as a result of the detailed study of the EDIF Version 300 and CFI DR 1.0 information models. Other topics which the study reviewed concerned the handling of properties and attributes (where EDIF supports inheritance through the instantiation mechanism and currently CFI DR does not), the representation of global signals/ports (e.g. clocks, power), the relevance or otherwise of the EDIF external library 
concept and issues related to the handling of strings (where EDIF supports a wide range of options through the ISO standards). Another topic that was reviewed concerns the CFI DR 2.0 plans to support an attachment operation. This is a means of attaching a cell (view) to a library (cell) other than the one in which it was initially defined. The mechanism proposed for CFI is very similar to the $\mathrm{UNIX}^{\mathrm{TM}}$ symbolic link. No definitive model was agreed for handling this in the core model and in a future version of EDIF, but it is clear that there are a number of possible ways to satisfy this requirement.

\section{Conclusions}

The real result of the study is that there are no technical 'show-stoppers' that prevent EDIF and CFI from coming closer together and sharing a common information model. The review has shown that the new ideas introduced into EDIF Version 300 as a result of the experience gained through the use of the EDIF Version 200 are fundamentally sound and are able to meet many of the needs of CFI for the future. The technical evidence indicates that the core model would be largely based on the EDIF Version 300 information model, possibly with some minor adjustments.

However, whether or not the step of creating the common core model will actually be taken depends less on technical issues than on practical ones of man power investment and of commercial and political will. The EDAC Tiger Team generally found the proposals acceptable so the issue now is how to effect the CFI transition to a common model. In practice, the move will probably be phased via an intermediate stage where the new core model is supported together with mappings to the existing CFI model via 'helper' functions. For EDIF, little or no change is required to meet the constraints of a common model. Minor alterations, particularly those not involving changes to the current syntax, would probably be acceptable even for EDIF Version 300 implementations if they were agreed quickly enough. Other changes, if agreed, could be included in the future PCB/MCM version of EDIF planned for release in 1995.

\section{References}

[1] Electronic Industries Association. Electronic Design Interchange Format Version 30 , EIA-618 December 1993 (4 volumes)

[2] CAD Framework Initiative Inc. CFI 1.0 Design Representation Programming Interface 1992

[3] ISO TC184/SC4/WG5 "EXPRESS Language Reference Manual" ISO 10303: Part 11 Version N14 April 1991

[4] Alan Williams, "Mapping between CFI 1.0 and EDIF Version 30 0", Version 0.1 Internal Report, CAD Group, Department of Computer Science, University of Manchester, UK, 1994

\section{Acknowledgements}

First of all, I would like to recognise the contribution made by Laurence Brevard of CFI to the work described here. We both learned a great deal about EDIF and CFI during our meetings and Laurence ensured that we maintained a focus on practical issues and drew on the expertise of the many people involved in creating and implementing EDIF and CFI. The interpretations presented here are, I believe, those that Laurence and I share. Thanks are also due to Alan Williams, Rachel Lau and Nigel Whitaker of the University of Manchester and Robin La Fontaine (Monsell) for advice and comments and to Don Cottrell (CFI), Rich Goldman (Synopsys and EDAC), Jeri Crowley (TI and EDIF) and Ron Christopher (IBM) for making sure that Laurence and I actually held the meetings. The financial support of the CEC through the ESIP Project (ESPRIT 8370) is gratefully acknowledged. 